\title{
Intelligent Car Park Management System using Wireless Sensor Network
}

\author{
Manisha Bhende \\ Research Center, Dypiet \\ University of Pune
}

\author{
Sanjeev Wagh \\ Research Center, Dypiet \\ University of Pune
}

\begin{abstract}
Parking problems are a common problem in most major cities. The limited availability of parking space results in traffic congestion, air pollution, time consuming as well as economy of the nation. The price for parking expansion is usually prohibitively or extremely high. All above various problems can be overcome by constructing a parking area that is embedded with Wireless Sensor Network Technology. Car-park management systems operate by monitoring the availability of car-parking spaces and making that information Available to customers and facility administrators. The prototype contains system architecture, software, hardware including its implementation phase. Our goal is to construct a car park system which is equipped with sensors and provides surveillance. We are developing a novel miniaturized modular platform for wireless sensor networks. The system architecture, hardware and software will be discussed as well as details of the deployment scenario chosen for the prototype of a car park management system.
\end{abstract}

\section{Keywords}

Infrared Sensor (IR), Wireless sensor networks (WSN), Radio frequency identification tag(RFID tag), Radio frequency reader(RF Reader), Liquid crystal Display (LCD).

\section{INTRODUCTION}

The Car parking is a parking area that utilizes various technologies to efficiently manage the lot early system used to display to drivers parking information such as the availability status and / or the number of available spaces.This system is still widely used today and now became the necessary part for building car parking area in major cities[1][2]. There are also complex car parking area that incorporates more advanced technologies to serve customers of different needs. Due to car park management system using wireless sensor network various problems like air pollution, time consumption and mainly the traffic congestion can be overcome to a great extent and this help peoples to park their car efficiently without an extra overhead.

Car-park management systems operate by monitoring the availability of car-parking spaces and making that

information Available to customers and facility administrators. Customers Use it for guiding them in their choice of parking space; administrators use it to aid in overall management and planning[3]. Sensor networks are a natural candidate for carpark management systems, because they allow status to be monitored very accurately-for each parking space, if desired. Wireless sensor networks have the advantage that they can be deployed in existing car-parks without having to install new cabling for network and electricity to reach each sensing device. For this reason, wireless sensor networks also have use for road-side car-parking.
The system contains RFID Tag and RFID Reader that strongly provide the security for the purpose of authentication of valid user to park the car. Monitoring system is used to save the details data of each individual who is parking his/her car in parking area. A car parking system is a mechanical device that has multiplies parking capacity to park the car inside a parking area. There are two types of car parking systems[4]: traditional and automated. In the long term, automated car parking systems are likely to be more cost effective when compared to traditional parking garages. Automatic car park systems are less expensive per parking lot, since they tend to require less building space and less ground area than a conventional facility with the same capacity[3][5].

\section{BACKGROUND}

\section{A Field of the Innovation}

Innovation relates to the energy conservation of IR sensor by making use of solar battery instead of using a regular power supply. Solar battery helps in energy conservation of IR sensor to a great extent. Whenever the car gets into parking area only the energy of that IR Sensor will be consumed on which the car is getting park and rest of the IR Sensor will be in the halt state. Hence, its energy will get conserve. Finding the lot for parking the car is done by calculating distance from the entry point of parking to the nearest parking lot in the parking area.

\section{Description of the Related Art}

This disclosure relates generally to the area of wireless sensor network and more specifically to car park management system using wireless sensor network. This revelation relates generally to the field of Wireless Sensor Network. Wireless Sensor Network emphasis on security networks, monitoring and recording the physical conditions of the environment and systemizing the collected data at a central location. The prototype works for monitoring or tracking of cars personnel or other physical conditions, for example in company for monitoring and control system.

Wireless Sensor Network encompasses a huge number of sensor nodes that have the ability to interact among themselves and also to external base station. Each sensor nodes have the processing power, limited sensing region and energy, networking a numerous nodes gives rise to a reliable, robust and precise sensor network covering a larger region.

Sensor network have number of real time application such as border surveillance, medical care, company monitoring ,fire monitoring, and highway traffic co-ordination ,military observation ,biomedical monitoring and industrial monitoring .In many of such application it is not possible or inconvenient to connect sensors by wires or by cables there for wireless network is preferred on a large scale Sensors are enlightened devices that are basically used to detect and respond to electrical or optics signals. A Sensor converts the physical 
parameter such as humidity, wind, speed, temperature, blood pressure, etc. into a signal which can be measured electrically.

Wireless network of sensor station however presents various technical challenges In comparison with the wired communication for the small distance, wireless communication generally requires higher power than wired communication. But in the case of individual wireless sensor station it requires a limited, self-contained power supply Wireless communication is vulnerable to congestion or unintentional interference. It is also open in that the source of transmission can be pointed by a hostile agent. If the data processing and control occurs at the central location then the whole network is vulnerable to the loss of central processor. Such a architecture has low dependency and cannot easily survive in an hostile environment.

Detection of car and personnel is more difficult than detecting large signals such as from earthquakes and moment of earth masses. For the small scale detection and personal infrared sensors are preferred Over ultra violet sensor because Infrared Sensor (IR) range is less than ultra violet sensor (UV) the reliable monitoring and tracking over large areas there by requires massive sensitive detector specially spaced closely .The emplacement of such massive conventional detectors is generally pricey, inconvenient and time consuming in case when they are wired for power supply or communication instead of that a wireless network is preferred as it have numerous sensitive, low powered sensor station and low cost.

\section{SYSTEM ARCHITECTURE AND DESCRIPTION}

The invention is a highly integrated, the miniature electronic sensing station, which can be used by other sensing station in a wireless communication network. The network and the sensor are basically used for sensing events such as car moment, intruders or any physical change or condition which can be detected by the sensors. Car Park management systems operate by a supervisor that checks the availability of car parking spaces and make that information available customers and facility administrators[1][2]. Customers use this information for finding the vacant parking space in parking areas, administrators use it for overall management and planning. Sensor networks are a natural candidate for car-park management systems, because they monitored the status very accurately-for each parking space.



Figure 1(a). System Architecture

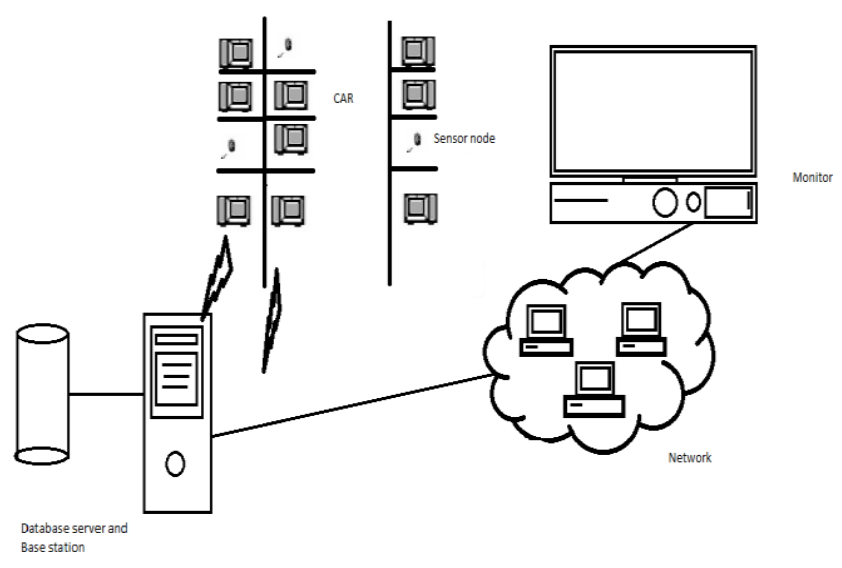

Figure 1(b) 3 Tier Architecture

FIG 1(a) shows the car parking system architecture which is encompassed by several components such as RFID Tag, RFID Reader, Solar battery, Microcontroller PIC16F877A, LCD LM016L, MAX232PTH Pin, parking area containing 4 parking lots equipped by IR Sensors. The RFID Tag is given to each user for authentication to check the valid user. This RFID Tag is being read by the RFID Reader which does the validation of the tag. After authentication the LCD which is of 14 pin will display the vacant parking lot to the user. The microcontroller is used to control and transfer the data. Transfer of data is done from computer via RS232 Cable and the data is given to microcontroller through the MAX232PTH pin which is used for data transfer as well as for voltage adjustment because the voltage of computer and the solar battery are different so to adjust them MAX232PTH Pin is used. The park areas have four parking lots and each one is equipped by sensors. For each parking lot there are two sensors one is transmitter and another one is receiver. As Sensors are the natural candidate of car park system it does the work of sensing. Instead of using a regular power battery solar battery is being used which greatly helps in conserving the energy of the sensors. Only the energy of that sensor will be consumed on which car is being park and rest of the sensors will be in halt state due to which energy will be conserved.

FIG 1 (b) Shows perspective view of three-tier architecture in which Tier 1 is used as monitoring system in which database will be stored of each and every employee and the higher layer shows the car park area equipped with sensors use by the users to park the car. Sensing and processing will be handling by tier 2 and the complete information sent by sensors stored in database server that is tier 3 in our architecture. Administrators do management and planning. The admin will store the detail information of the user and keep on updating the information. According to the availability of the park lot car will be parked. Customers park the car at vacant parking space in parking area. Sensor networks are a natural candidate for car-park management systems, because they monitored the status very accurately-for each parking space. 


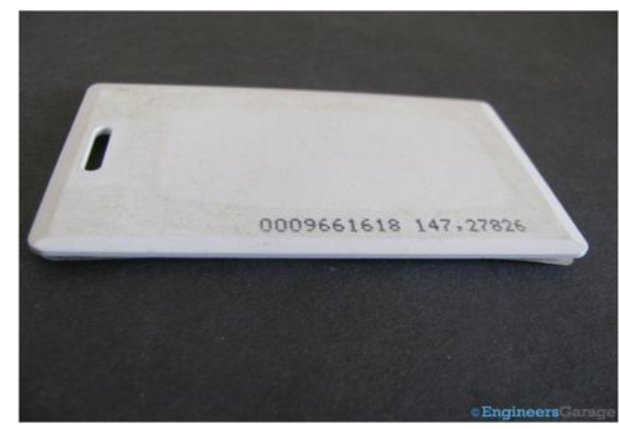

Figure 2.RFID Tag



Figure 3. 2internal structure of RFID Tag



Figure 4 RFID Reader

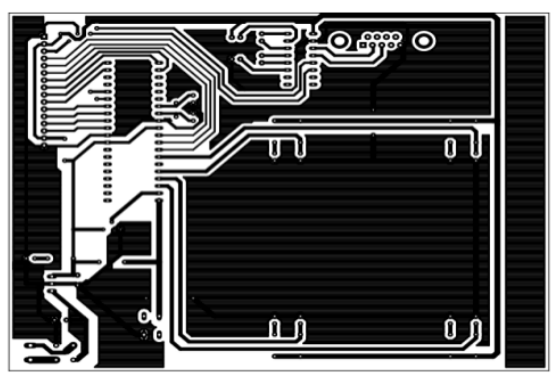

Figure 5 Soldering

FIG 3.Shows the internal structure of Radio Frequency Identification [RFID] Tag. The RFID Tag has a small chip at center and a golden coil is surrounded over it. This chip store and transmit information. Stored information is read out by the RFID reader. The RFID reader's function is to interrogate RFID tags[3].

FIG 4.Shows a snapshot of Radio Frequency Identification [RFID] Reader. It is a radio frequency transmitter and receiver that communicate with tags. Reader using attached signal, receive data from the tag and then pass it to computer system for processing via RS232 Cable. The main task of RFID Reader is validation[4]. RFID Tag validation is done by RFID Reader. RFID Reader greatly helps in providing security.
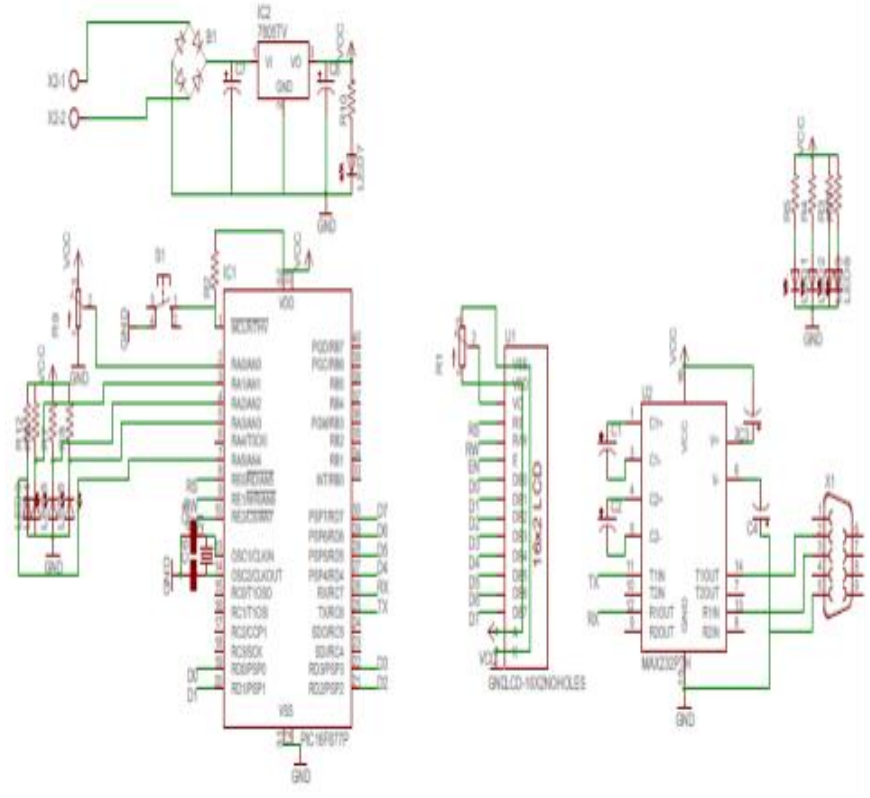

Figure 6. Internal Circuitry

FIG 6.Shows perspective view of internal circuitry of the system in which connections between microcontroller PIC16F877A, MAX 232PTH and LCDLM016L are established and registers are used to regulate voltage. Microcontroller PIC16F877A is a 8 bit microcontroller which is having 3 ports that is used for input output configuration. The pins RD0 to RD7 are connected to the data port of LCD that is D0 to D7.These pins are output pins and also the unidirectional pins.

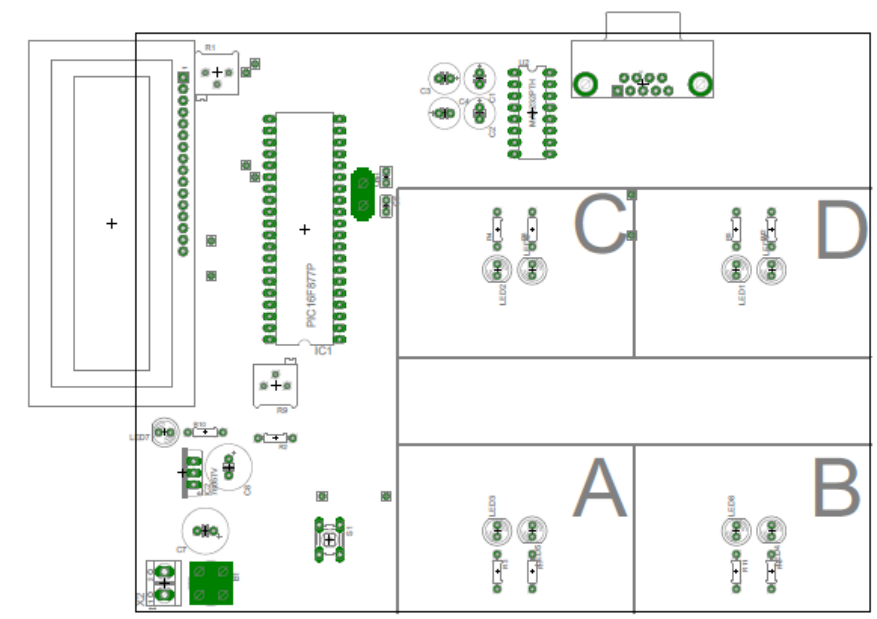

Figure 7 . Internal Layout of Car park area

FIG 7 .Shows the Perspective internal sketch layout of car park system. The diagram shows the placement of the components. Roughly the sketch layout of internal car park system is done and according the prototype will be design for the system. 


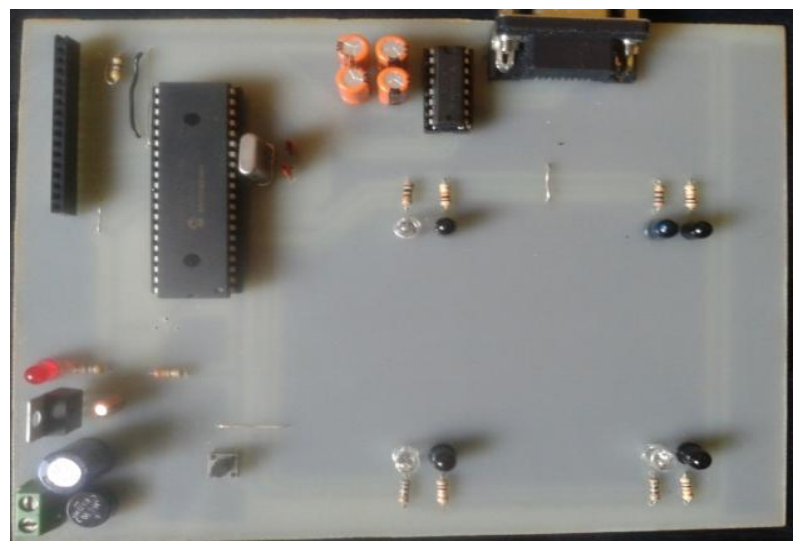

Figure 8. Outer layout of Car Park area

FIG 8 . Shows implementation circuit of all components used in Car Parking Management System using Wireless Sensor Network. In which Microcontroller PIC16F877A automatic controlling device for IR sensor, RS232.it is selfreprogrammable under software control and having power saving sleeping mode. MAX232PTH is used for serial communication of data. It is also help to adjust voltage power between system and circuit. The wireless sensor node detects the status of parking space with IR sensor and transmits or receives messages. Register stores the data. LED will Displays the available parking area at entry gate Radio Frequency Identification (RFID) is an emerging technology that is increasingly being used in business and industry. The RFID tag contains electronically store information which is read up by the RFID Reader for security[5].

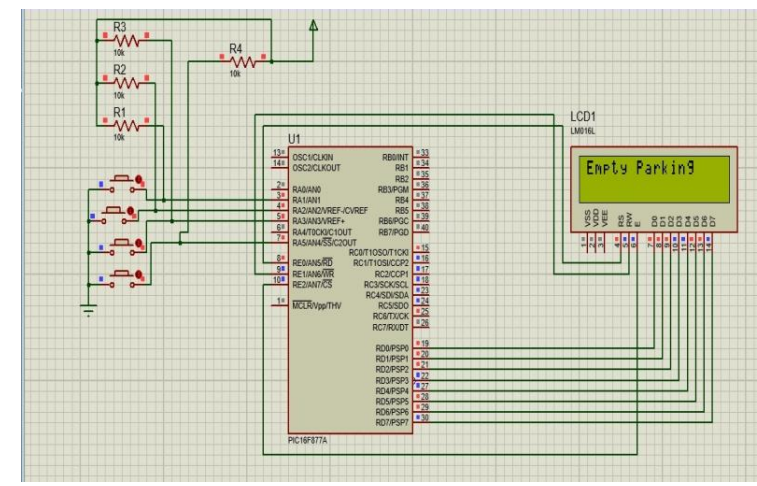

Figure 9. PCB layout

FIG 9.Shows the snapshot of Peripheral circuit board [PCB] of the car park system. The diagram reflect the view of the system when lot A is occupied and rest of B,C,D lots are empty which are display on LCD. When a car get park at lot A in a parking area then sensors generates the signal and sends that generated signal to controller and then LCD will display the updated available parking lots in parking area. The A parking lot sensor is pressed that means A parking lot is allotted so the information will be updated and the LCD will show the remaining vacant parking lots to the user.

\section{SYSTEM DESIGN AND SOFTWARE IMPLEMENTATION}

The software required for the car park management project is java and c language. The system must contain turbo $\mathrm{C}$ setup and the net beans to run the project. Java is a programming language originally developed at Sun Microsystems and released in 1995 as a core component of Sun Microsystems' Java platform. The language derives much of its syntax from C and $\mathrm{C}++$ but has a simpler object model and fewer low-level facilities. Java applications are typically compiled to byte code (class file) that can run on any Java Virtual Machine (JVM) regardless of computer architecture. Java is a general-purpose, concurrent, class-based, object-oriented language that is specifically designed to have as few implementation dependencies as possible. It is intended to let application developers "write once, run anywhere" (WORA), meaning that code that runs on one platform does not need to be recompiled to run on another. Java is currently one of the most popular programming languages in use, particularly for client-server web applications, with a reported 10 million users. The original and reference implementation Java compilers, virtual machines, and class libraries were developed by Sun from 1995. As of May 2007, in compliance with the specifications of the Java Community Process, Sun relicensed most of its Java technologies under the GNU General Public License. Others have also developed alternative implementations of these Sun technologies, such as the GNU Compiler for Java and GNU Class path.

C language is used for hardware. The coding of IR Sensors and registers is done in $\mathrm{C}$ language which helps to connect the hardware and the GUI which help out to execute the system.

\subsection{Databases:}

A database is a structured collection of data. It may be anything from a simple shopping list to a picture gallery or the vast amounts of information in a corporate network. To add, access, and process data stored in a computer database, you need a database management system such as MS access. Since computers are very good at handling large amounts of data, database management systems play a central role in computing, as standalone utilities, or as parts of other applications. 


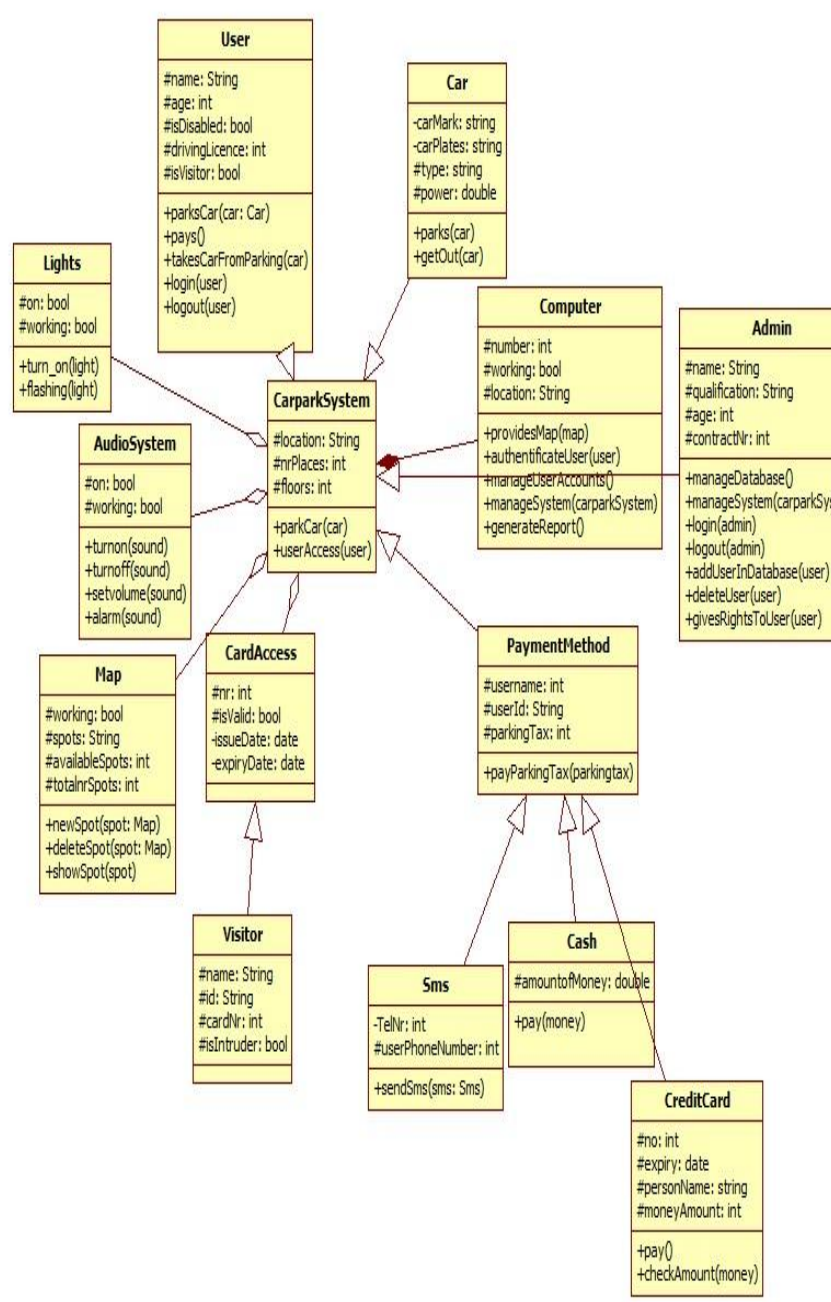

Figure 10. Advance class diagram

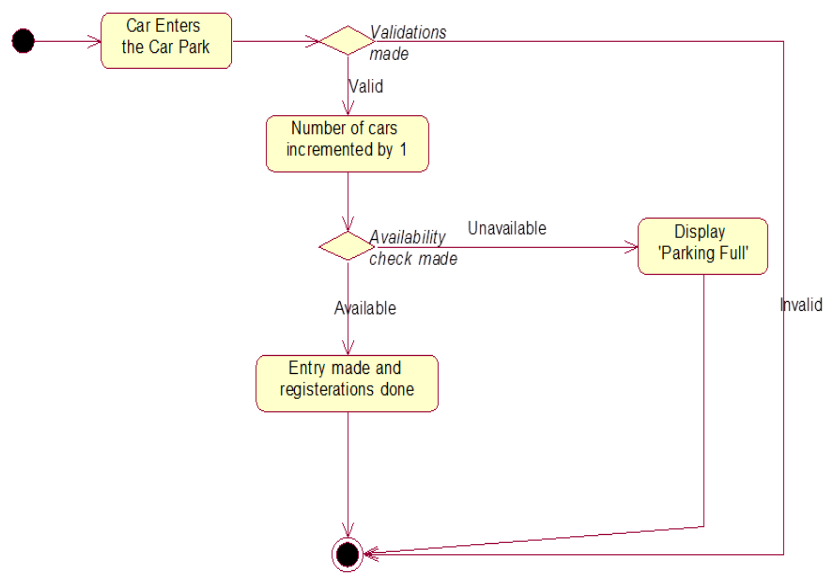

Figure 11. Activity diagram

MS access databases are relational. A relational database stores data in separate tables rather than putting all the data in one big storeroom. The database structures are organized into physical files optimized for speed. The logical model, with objects such as databases, tables, views, rows, and columns, offers a flexible programming environment. Java can be connected to MS access by JDBC ( Java Database Connectivity ).
MS access is used to store the confidential data of the user along with its details. The database is handled and managed by the admin. The database stored are name, Tag number, address, contact information, car plate number, entry time, exit time etc.

\subsection{Important modules and algorithm}

The project has number of hardware modules:

System Architecture embodies with various modules such as RFID Tag, RFID Reader, Microcontroller PIC16F877A, LCD LM016L, IR Sensor and Solar Battery.

1.RFID Tag: The RFID Tag used for authentication. FID Tag contains a chip at the centre and the coil field is surrounded over the chip.

2.RFID Reader: The RFID Reader which is use to read the RFID Tag. The Detection of authorized Tag is done by RFID Reader.

3. LCD: The Liquid Crystal Display [LCD] LM016L which is used to display the vacant parking lot.

4.Microcontroller: The microcontroller PIC16F877A is a 40 pin package; 8 bit microcontroller is used for controlling automatic devices.

5.MAX232PTH Pin: The MAX232PTH chip which used for data transfer as well as voltage adjustment.

6. IR Sensor: The Infrared [IR] Sensor which are used for sensing the car in parking area.

7. RS232 Cable: Two RS232 Cable are used one for the connection between computer and the hardware and second for the connection between computer and RFID Reader.

\section{RESULTS}

- Representing initial empty car park area

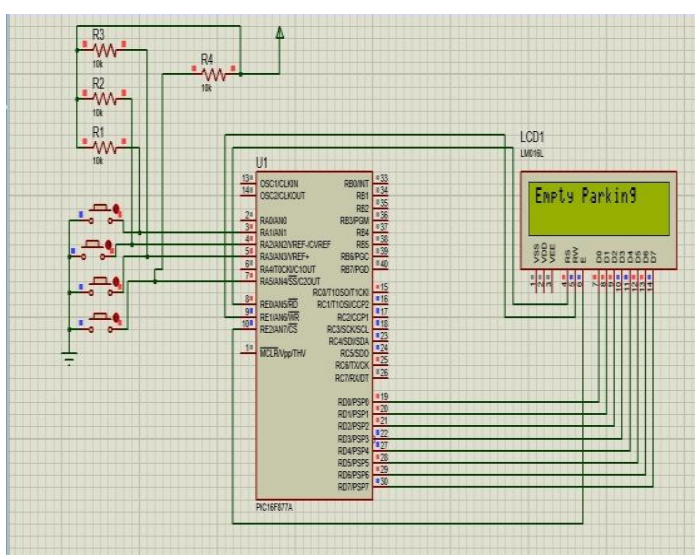

- Representing the car park area where A slot is accommodated and rest of B,C,D slots are empty. 


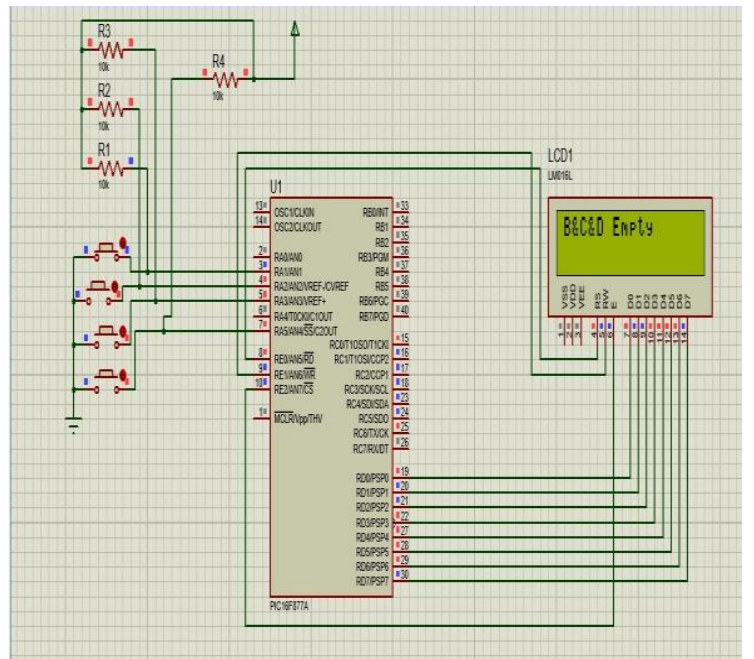

- $\quad$ Representing the car park area where A and B slot is accommodated and rest of C,D slots are empty.

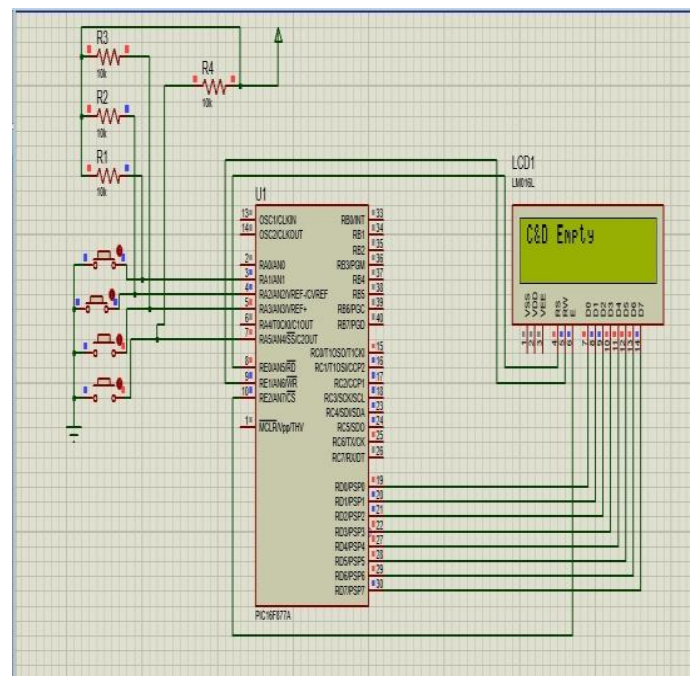

\section{CONCLUSION}

Smart parking systems employ advanced technologies to permit efficient use of parking lots. Smart parking ranges from simple systems that show the number of available spaces to complex ones that can guide customers to a free spot. In addition, smart parking may incorporate transit based information, smart payment, and auto-parking to best serve customers' various needs. In most smart parking, vehicle detectors are an indispensable component. We proposed to employ optical sensor in WSN to replace commonly used loop detectors. Optical WSN allowed for easy installation and maintenance with the same or better accuracy. Our original WSN was modified to include two optical sensor heads. With two sensor heads, vehicles can be distinguished from pedestrians as well as motorcycles. The vehicle direction information can also be obtained with two sensor heads. We plan to implement this optical WSN at our main parking lot and test for its workability.

\section{REFERENCES}

[1] J. P. Benson, t. O'donovan, p. O'sullivan, u. Roedig, c. Sreenan university college CORK (ucc), cork, ireland "car-park management using wireless sensor networks"

[2] Joseph jeffrey, roshan gajanan patil, skanda kumar kaipu narahari, yogish didagi, jyotsna bapat, debabrata das international institute of information technology, bangalore "smart parking system using wireless sensor networks" copyright (c) iaria, 2012. Isbn: 978-1-61208-207-3.

[3] Mala aggarwal, simmi aggarwal, r.s.uppal "comparative implementation of automatic car parking system with least distance parking space in international journal of scientific and research publications, volume 2, issue 10, October 2012 1issn 2250-3153 wireless sensor networks" Gartner Report, Financial Times, 2007.

[4] Mingkai Chen, Tainhai Chang, "A Parking guidance \& Information based on Wireless Sensor Networks", IEEE International Conference on information \& Automation Shenzhen, China, June 2011.

[5] Abhijit Sharma, Rituparna Chaki, Uma Bhattacharya, "Applications of Wireless Sensor Network in intelligent traffic system: A Review", 978-1-42448679-3/11,IEEE 2011.

[6] J. Chinrungrueng, U. Sunantachaikul, and S.

[7] Triamlumlerd.A vehicular monitoring system with power-efficient wireless sensor networks. In 6th International Conference on ITS Telecommunication Proceedings, pages 951-954. IEEE Press, 2006. L. Klein. Sensor Technologies and Database

[8] Requirements for ITS. Artech House, 2001. S.Shaheen,C.Rodier,andA.Eaken.Smartparking management field test :Abayarearapid transit(bart)district arking demonstration,Jan2005. Final Report. 